\title{
BMJ Open Cross-sectional study of diet, physical activity, television viewing and sleep duration in 233110 adults from the UK Biobank; the behavioural phenotype of cardiovascular disease and type 2 diabetes
}

\author{
Sophie Cassidy, ${ }^{1}$ Josephine $\mathrm{Y}$ Chau, ${ }^{2}$ Michael Catt, ${ }^{3}$ Adrian Bauman, ${ }^{2}$ \\ Michael I Trenell ${ }^{1}$
}

To cite: Cassidy S, Chau JY, Catt $\mathrm{M}$, et al. Cross-sectional study of diet, physical activity, television viewing and sleep duration in 233110 adults from the UK Biobank; the behavioural phenotype of cardiovascular disease and type 2 diabetes. BMJ Open 2016;6:e010038. doi:10.1136/bmjopen-2015010038

- Prepublication history and additional material is available. To view please visit the journal (http://dx.doi.org/ 10.1136/bmjopen-2015010038).

Received 18 September 2015 Revised 15 January 2016 Accepted 29 January 2016

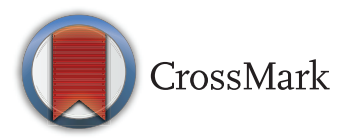

For numbered affiliations see end of article.

Correspondence to Professor Michael Trenell; michael.trenell@ncl.ac.uk

\section{ABSTRACT}

Objectives: Simultaneously define diet, physical activity, television (TV) viewing, and sleep duration across cardiometabolic disease groups, and investigate clustering of non-diet lifestyle behaviours.

Design: Cross-sectional observational study.

Setting: 22 UK Biobank assessment centres across the UK.

Participants: 502664 adults aged 37-63 years old, $54 \%$ women. 4 groups were defined based on disease status; 'No disease' ( $\mathrm{n}=103$ 993), 'cardiovascular disease' (CVD $n=113$ 469), 'Type 2 diabetes without CVD' $(n=4074)$ and 'Type 2 diabetes + CVD' $(n=11574)$.

Main outcomes: Diet, physical activity, TV viewing and sleep duration.

Results: People with 'CVD' report low levels of physical activity $(<918 \mathrm{MET} \mathrm{min} /$ week, OR $(95 \% \mathrm{Cl})$ 1.23 (1.20 to 1.25)), high levels of TV viewing ( $>3 \mathrm{~h} /$ day; 1.42 (1.39 to 1.45)), and poor sleep duration $(<7$, $>8 \mathrm{~h} /$ night; 1.37 (1.34 to 1.39)) relative to people without disease. People with 'Type 2 diabetes + CVD' were more likely to report low physical activity (1.71 (1.64 to 1.78)), high levels of TV viewing (1.92 (1.85 to 1.99)) and poor sleep duration (1.52 (1.46 to1.58)) relative to people without disease. Non-diet behaviours were clustered, with people with 'CVD' or 'Type 2 diabetes + CVD' more likely to report simultaneous low physical activity, high TV viewing and poor sleep duration than those without disease (2.15 (2.03 to 2.28 ) and 3.29 (3.02 to 3.58), respectively). By contrast, 3 in 4 adults with 'Type 2 diabetes', and 2 in 4 adults with 'CVD' have changed their diet in the past 5 years, compared with only 1 in 4 in the 'No disease' group. Models were adjusted for gender, age, body mass index, Townsend Deprivation Index, ethnicity, alcohol intake, smoking and meeting fruit/vegetable guidelines.

Conclusions: Low physical activity, high TV and poor sleep duration are prominent unaddressed high-risk

\section{Strengths and limitations of this study}

- Diet, physical activity, television (TV) viewing and sleep duration were simultaneously investigated in a very large and well-described UK population cohort.

- Cardiometabolic disease groups were compared with disease-free individuals, and show that worsening cardiometabolic health is associated with a progressive unhealthy behavioural phenotype, consisting of low physical activity, high TV viewing and poor sleep duration.

- The cross-sectional nature means we cannot infer causality between disease and lifestyle behaviours.

- Lifestyle behaviours were self-reported, which may limit the accuracy of measurements.

characteristics of both CVD and type 2 diabetes, and are likely to be clustered together.

\section{INTRODUCTION}

Cardiovascular disease (CVD) and type 2 diabetes represent significant personal, economic and societal burdens. CVD accounts for a quarter of all UK deaths, ${ }^{1}$ and people with type 2 diabetes carry twice the risk of developing CVD. ${ }^{2}$ With over 700 new cases of diabetes diagnosed daily, ${ }^{3}$ total healthcare expenditure on diabetes is forecast to rise from $10 \%$ to $17 \%$ by $2035 .{ }^{4}$ The interrelationship between cardiovascular and metabolic disease is termed cardiometabolic health, and reflects their common environmental and genetic antecedents. Those with both CVD and type 2 diabetes have a 
particularly poor prognosis, and require aggressive risk factor intervention. ${ }^{5}$

Behavioural factors, spanning diet, physical activity, sedentary behaviour and sleep are major risk factors for the development of cardiometabolic disease. The reduction in energy expenditure through (1) lack of physical activity and (2) increase in sedentary behaviours are risk factors for cardiometabolic disease. ${ }^{6} 7$ Indeed, technological advancements of the 21st century have paved the way for sedentary behaviours, such as watching TV, driving and sitting at a computer becoming the 'norm' in modern society, so that physical inactivity is now the fourth leading cause of disease and disability in the $\mathrm{UK}^{8}$ An important lifestyle behaviour, but often forgotten, is sleep, and this is strongly linked to cardiometabolic disease. ${ }^{9}{ }^{10}$ Sleep is vital for resetting homoeostasis and regulating metabolism, yet changes in working patterns and increased demands on time means sleep debt is a growing issue.

Since WHOs global strategy on diet, physical activity and health, ${ }^{11}$ there have been calls for countries to develop national policy approaches to these lifestyle behaviours. ${ }^{12}$ Indeed, in 2011 the UK government published physical activity recommendations, ${ }^{13}$ and Eat Well was produced as a policy tool that defines government recommendations on healthy diets. ${ }^{14}$ Specific National Institute for Health and Care Excellence (NICE) recommendations for CVD and type 2 diabetes recognise the importance of improving physical activity and diet, but guidance on sitting time or sleep behaviours has not been addressed. ${ }^{15}{ }^{16}$ Nonetheless, knowledge of baseline behaviours in the population are lacking.

The UK Biobank is a large population-based cohort, and allows measurement of important lifestyle behaviours at the same time, at scale and in a well-described cohort. Our primary aim was to observe the differences in lifestyle behaviours simultaneously across cardiometabolic disease. Our secondary objective was to explore clustering of unhealthy non-diet behaviours across disease groups.

\section{METHODS}

\section{Population and study design}

A cross-sectional analysis was conducted on baseline data from the UK Biobank. The UK Biobank is a large, population-based cohort study examining the interrelationships between environment, lifestyle and genes. ${ }^{17}$ Around 9.2 million invitations were mailed to recruit 502664 adults (response rate 5.5\%) aged between 37 and 73 years. ${ }^{17}$ Recruitment occurred between 2007 and 2010, via 22 assessment centres across the UK. During an assessment centre visit, there were six stages; consent, touchscreen questionnaire, verbal interview, eye measures, physical measures and blood/urine sample collection. The touchscreen questionnaire covered sociodemographics, occupation, lifestyle, early life exposures, cognitive function, family history of illness and medical history. Details of procedures have been previously published. ${ }^{17}$ Participant written informed consent was obtained prior to data collection. All data extracted were deidentified for analysis.

\section{Disease categories}

Self-report disease status was obtained from participants during the touchscreen questionnaire, which was then entered and verified by a UK Biobank nurse after further questioning during the verbal interview. We have identified four disease groups spanning cardiometabolic health. (1) Healthy reference group: Participants with no disease listed were classified as the 'No disease' group. (2) CVD: Based on the International Classification of Diseases $10^{18}$ and a clinician's opinion (AB), diseases to include in the 'CVD' group were selected and any patients with type 2 diabetes or diabetes-related comorbidities (including diabetic neuropathy, diabetic nephropathy and diabetic eye disease) were excluded (a list of diseases included in the CVD group can be found in the online supplementary material 1). Type 2 diabetes: Participants who were entered as having 'diabetes' or 'type 2 diabetes' were selected. Those taking insulin within their first year, and were $<35$ years old at diagnosis were excluded to reduce the likelihood of type 1 and monogenic forms of diabetes. Those without and with CVD were separated into (3) 'Type 2 diabetes without CVD' and (4) 'Type 2 diabetes + CVD', respectively (figure 1). Excluded from analysis was the 'Other diseases' group $(n=203700)$ with a wide range of diseases covering respiratory, gastrointestinal, renal, neurology, musculoskeletal, haematology, gynaecology, immunological and infectious. A general summary of this group can be found in online supplementary material 2.

\section{Baseline measurements}

Sociodemographic, smoking, alcohol and dietary intake, physical activity, TV viewing and sleep duration data were collected from the touchscreen questionnaire. Physical activity was assessed using adapted questions from the validated short International Physical Activity Questionnaire $(\mathrm{IPAQ})^{19}$ which covers the frequency, intensity and duration of walking, moderate and vigorous activity. Time spent in vigorous, moderate and walking activity was weighted by the energy expended for these categories of activity, to produce MET min/ week of physical activity, which is referred to as 'total physical activity'. Data processing rules published by IPAQ were followed. ${ }^{20}$ MET.min/week was used for further analysis across disease groups in this manuscript, but we have also included analysis of the separate frequency (days) and duration (min) in online supplementary material 3 .

To measure TV viewing, participants were asked 'In a typical day, how many hours do you spend watching television?' based on previous literature. ${ }^{21}$ This was asked twice to those who responded $>8 \mathrm{~h}$, therefore, high 


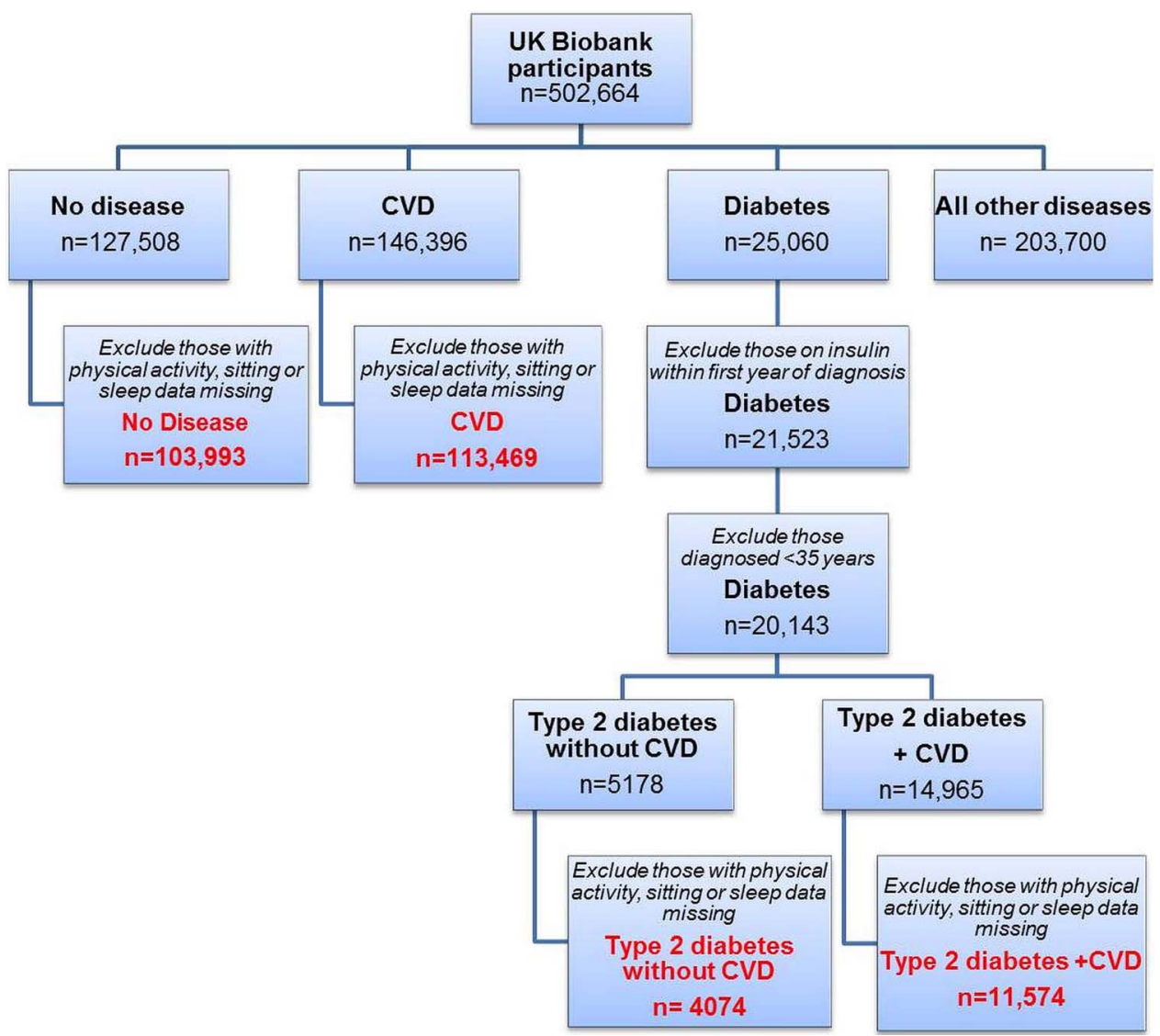

Figure 1 Flow chart demonstrating how disease groups were defined. Final four groups shown in red.

values were deemed robust. To measure sleep duration, participants were asked 'About how many hours sleep do you get in every $24 \mathrm{~h}$ ? (please include naps)'. This was asked twice to those who responded $>12 \mathrm{~h}$.

Diet intake was reported using the Food Frequency Questionnaire, ${ }^{22}$ in which a number of questions were used based around commonly eaten food groups. Information on fresh/dried fruit, salad and cooked/raw vegetables were combined to create a binary variable to identify individuals who did and did not meet the UK's current guidelines on fruit and vegetable consumption (5/day). ${ }^{14}$ Participants were asked 'Have you made any major changes to your diet in the last 5 years?' and were also required to select any of the following foods they 'Never eat'; eggs, dairy, wheat or sugar. This data was used to observe dietary change and sugar consumption across the four disease groups.

Townsend Deprivation Index was used as a measure of socioeconomic status, by combining census data and postal codes of participants. The index combines information on housing, employment, car availability and social class, with higher values indicating greater deprivation.

Body mass index (BMI) was calculated from: weight $(\mathrm{kg}) /$ height $(\mathrm{m})^{2}$. Weight was measured using the Tanita BC-418MA body composition analyser, to the nearest $0.1 \mathrm{~kg}$ and height was measured using a Seca 202 height measure. Trained staff took these measures, and participants were required to remove shoes and heavy outer clothing.

\section{Statistical analysis}

All data analyses were performed using SPSS, V.21.0 (IBM, Armonk, New York, USA). Individuals with missing data on total physical activity, TV viewing or sleep duration, were excluded (figure 1). Online supplementary material 4 shows the sociodemographics of missing cases, which were similar to the main cohort, but had a lower proportion of men across all groups. Total physical activity, vigorous, moderate and walking minutes alongside TV viewing were categorised into four groups based on the quartile demarcators for the "no disease' group. Total physical activity groups were labelled as 'low physical activity' (lowest quartile: $\leq 918$ MET.min/week) and 'high physical activity' (highest quartile: >3706-19 278 MET.min/week) and TV viewing was labelled as 'low TV viewing' (lowest quartile: $\leq 1 \mathrm{~h}$ / day) and 'high TV viewing' (highest quartile: $>3 \mathrm{~h} /$ day). As sleep duration shows a 'U'-shaped relationship with diabetes risk (rather than a linear relationship like physical activity and TV viewing), the data were split using predefined thresholds from the literature; $<7 \mathrm{~h}, 7-8 \mathrm{~h}$ and $>8 \mathrm{~h}$ cut points were used based on a recent meta-analysis. ${ }^{10}$ Sleep duration was labelled as "poor sleep duration' ( $<7$ or $>8 \mathrm{~h} /$ night) and 'good sleep duration' (7-8 h/night). Owing to the large sample size, 
Pearson's $\chi^{2}$ deemed any small difference in group proportions as significant, therefore, these results are not reported.

Non-diet lifestyle behaviours (including physical activity, TV viewing and sleep duration) were further analysed across cardiometabolic disease groups. The proportion of adults reporting all three unhealthy non-diet lifestyle behavoiurs (low physical activity, high TV viewing and poor sleep duration) was calculated for each group and labelled as having an 'unhealthy phenotype'. By contrast, those who reported high physical activity, low TV viewing and good sleep duration were labelled as having a 'healthy phenotype'. Binary logistic regression was used to determine the odds of reporting low physical activity, high TV viewing and poor sleep separately, alongside the odds of reporting an 'unhealthy phenotype' across disease groups. Additionally, to further investigate the importance of physical activity intensity in cardiometabolic disease, binary logistic regression was used to compare the odds of reporting low walking, moderate and vigorous activity across disease groups. Adjusted ORs, with 95\% CIs were reported. All logistic regression models were adjusted for: age (reference $={ }^{\prime} 40-49$ '); gender (reference $=$ 'Female'); BMI (reference $={ }^{\prime}<18.5-$ 24.9'); Townsend Deprivation Index (reference='least deprived); ethnicity (reference='White/British); alcohol $($ reference $=$ 'Never'); smoking $\quad($ reference $=$ 'Never'); meets fruit/vegetable guidelines (reference='YES'). Of the 233110 cohort, data was missing for; BMI $(0.006 \%)$,
Townsend Deprivation Index $(0.002 \%)$, ethnicity $(0.003 \%)$, smoking status $(0.003 \%)$, alcohol status $(0.001 \%)$, and fruit and vegetable guidelines $(0.015 \%)$, therefore, these cases were excluded from the logistic regression models. All statistical tests were two-sided, and significance was set at $\mathrm{p}<0.05$.

\section{RESULTS}

Of the 502664 UK Biobank participants, after excluding those with missing data or who were likely to have type 1 diabetes, there were 103993 (21\%) with no disease, 113469 (23\%) with CVD, $4074(1 \%)$ with type 2 diabetes without CVD, and $11574(2 \%)$ with type 2 diabetes + CVD (figure 1). As expected across worsening cardiometabolic disease groups, the proportion of men and those aged $>60$ years old increased, as did those classified as obese (table 1). There was a marked increase in obesity, with numbers almost quadrupling in the 'Type 2 diabetes + CVD' group, compared with disease-free individuals $(60.0 \%$ vs $15.0 \%)$. The 'No disease' group had a higher proportion of white/British, and least deprived individuals compared with cardiometabolic diseases. According to the Townsend Deprivation Index, socioeconomic status decreased across cardiometabolic disease groups (table 1).

Compared with the 'No disease' group, the 'Type 2 diabetes + CVD' group reported higher levels of previous smoking $(\mathrm{n}=5571(48.3 \%)$ versus $\mathrm{n}=30960(29.8 \%))$

Table 1 Sociodemographics characteristic of disease groups $(n=233110)$

\begin{tabular}{|c|c|c|c|c|}
\hline & \multicolumn{4}{|c|}{ Percentage within each disease group } \\
\hline & $\begin{array}{l}\text { No disease } \\
(n=103993)\end{array}$ & $\begin{array}{l}\text { CVD } \\
(n=113469)\end{array}$ & $\begin{array}{l}\text { Type } 2 \text { diabetes } \\
\text { without CVD }(n=4074)\end{array}$ & $\begin{array}{l}\text { Type } 2 \text { diabetes + } \\
\text { CVD }(n=11574)\end{array}$ \\
\hline \multicolumn{5}{|l|}{ Sociodemographics } \\
\hline$\%$ Male & 47.0 & 53.3 & 63.6 & 68.0 \\
\hline Age $(n)$, years & 103993 & 113469 & 4074 & 11574 \\
\hline $37-49$ & 35.6 & 12.5 & 13.5 & 6.0 \\
\hline $50-59$ & 35.9 & 30.2 & 32.6 & 27.6 \\
\hline $60-73$ & 28.6 & 57.4 & 53.9 & 66.4 \\
\hline BMI $(n), k g / m_{2}$ & 103443 & 112852 & 4048 & 11478 \\
\hline$<18.5-24.9$ (under and acceptable weight) & 42.9 & 22.0 & 14.9 & 7.2 \\
\hline 25-29.9 (overweight) & 42.1 & 44.4 & 40.5 & 32.8 \\
\hline$\geq 30$ (obese) & 15.0 & 33.6 & 44.6 & 60.0 \\
\hline Townsend deprivation quintile (\%) & 103861 & 113323 & 4070 & 11557 \\
\hline 1 (least deprived) & 21.9 & 19.7 & 17.7 & 14.7 \\
\hline 2 & 20.8 & 19.9 & 17.3 & 17.4 \\
\hline 3 & 20.7 & 19.9 & 18.9 & 18.5 \\
\hline 4 & 19.6 & 20.0 & 20.8 & 21.0 \\
\hline 5 (most deprived) & 17.1 & 20.5 & 25.1 & 28.5 \\
\hline Ethnicity (n) & 103687 & 113130 & 4060 & 11528 \\
\hline White/British & 94.6 & 95.0 & 85.6 & 89.9 \\
\hline Mixed & 0.6 & 0.5 & 0.6 & 0.6 \\
\hline Asian & 1.8 & 1.7 & 8.1 & 5.1 \\
\hline Black African & 1.5 & 1.8 & 3.3 & 2.9 \\
\hline Chinese & 0.5 & 0.2 & 0.5 & 0.2 \\
\hline Other & 0.9 & 0.7 & 2.0 & 1.3 \\
\hline
\end{tabular}


and alcohol $(\mathrm{n}=853(7.4 \%)$ versus $\mathrm{n}=2020(1.9 \%))$, but lower current alcohol consumption $(\mathrm{n}=9891 \quad(85.5 \%)$ vs $\mathrm{n}=98354(94.6 \%))$. Dietary data indicate that threequarters of those with type 2 diabetes have altered their diet within the past 5 years ('Type 2 diabetes without CVD': $75.5 \%$ and 'Type 2 diabetes + CVD': $75.3 \%$ ), and also half of them never eat sugar ('Type 2 diabetes without CVD': $49.8 \%$ and 'Type 2 diabetes + CVD': $51.2 \%$ ), which is proportionally more than the 'CVD' and 'No disease' groups. Around a third of the 'No disease' group met the UK's fruit and vegetable guidelines $(29.8 \%)$ with an increasing trend across cardiometabolic disease (table 2). All other dietary behaviours are reported in online supplementary material 5 .

Total physical activity levels declined across cardiometabolic disease groups (table 2 and figure 2). Vigorous activity was the main contributor to the reduction in total physical activity levels, with a smaller proportion of adults in the 'Type 2 diabetes + CVD' group reaching the upper quartile of vigorous activity compared with the 'No disease' group (12.3\% vs 23.7\%) (table 2). Those with 'Type 2 diabetes + CVD' were $34 \%, 55 \%$ and $80 \%$ more likely to report low walking, moderate and vigorous activity levels, respectively, compared with the 'No disease' group (table 3). The proportion of adults who reported high TV viewing more than doubled in the 'Type 2 diabetes + CVD' group compared with the 'No disease' group (47.3\% vs $20.3 \%$ ) (figure 2 ). These results indicate that almost half the adults diagnosed with 'Type 2 diabetes + CVD' sit for $>3 \mathrm{~h}$ /day watching TV. Almost three-quarters of the 'No disease' group report optimal sleep duration, but this proportion declined across cardiometabolic disease groups. The proportion of poor sleepers $(<7$ and $>8 \mathrm{~h})$ was higher in cardiometabolic disease groups compared with the "No disease' group (figure 2). The proportion of adults who reported an 'unhealthy phenotype' increased across worsening cardiometabolic disease groups from $1.8 \%$ (No disease) to $10 \%$ (Type 2 diabetes + CVD), and the proportion reporting a 'healthy phenotype' decreased across groups (table 2).

Those with the most serious cardiometabolic disease profile (Type 2 diabetes + CVD) were 70\% (OR (95\% CI) 1.71 (1.64 to 1.78$)$ ), $90 \%$ (1.92 (1.85 to 1.99$)$ ) and $50 \%$ (OR $1.52,95 \%$ CI 1.46 to 1.58 ) more likely to report low physical activity, high TV viewing and poor sleep duration, respectively, compared with the "No disease' group (table 4). The odds of reporting all three unhealthy behaviours together was higher than reporting one of these lifestyle behaviours individually. Indeed, those in the 'Type 2 diabetes + CVD' group were three times more likely to report an 'unhealthy phenotype', (ie, low physical activity, high TV viewing and poor sleep duration) (OR=3.29 (95\% CI 3.02 to 3.58)) even when controlling for age, gender, BMI, Townsend Deprivation Index, ethnicity, alcohol, smoking and meeting fruit/ vegetable guidelines. The shift in unhealthy behaviours is visualised in figure 3 which shows the movement from healthy behaviours (green/right) to unhealthy behaviours $(\mathrm{red} / \mathrm{left})$.

\section{DISCUSSION}

This is the largest cohort study to simultaneously assess diet, physical activity, TV viewing and sleep duration across cardiometabolic disease and non-disease groups. The results indicate that compared with disease-free individuals, (1) those with cardiometabolic disease report less physical activity, higher TV viewing and poorer sleep patterns; (2) non-diet unhealthy lifestyle behaviours were clustered, and people with 'Type 2 diabetes + CVD' were more than three times more likely to report low physical activity, high TV viewing and poor sleep duration at the same time and (3) people with cardiometabolic disease had changed their diet and were less likely to consume sugary foods. These results suggest that recommendations to change diet are reaching those with cardiometabolic disease, yet low physical activity, high TV viewing and poor sleep duration are significant, unaddressed, cardiometabolic risk factors.

\section{Strengths and weaknesses of the study}

The present report holds strength in the large sample size and detailed measurements. The population-based design allows simultaneous presentation of behaviours in people with different stages of cardiometabolic disease, controlling for key factors including age, sex, socioeconomic status and BMI. However, the study is not without limitation. The response rate of the UK Biobank was low $(5.5 \%)$, and a number of participants were excluded due to missing values, which may affect the generalisibility of the results to the UK population. The cross-sectional nature means we cannot infer whether these unhealthy lifestyle behaviours precede or were preceded by cardiometabolic disease. Over time, as the UK Biobank cohort progresses, longitudinal observations will be possible. Lifestyle behaviours were self-reported and not objectively measured. However, all questionnaires used validated self-reporting which allows these measures to be applied to large numbers of people. TV viewing is a poor surrogate for total daily sitting time, which is a more complete measure of sedentary behaviour, but could not be calculated from the UK Biobank questionnaire used. Residual confounding has to be acknowledged, as TV viewing is also influenced by other unhealthy behaviours such as increased snacking. ${ }^{23}$ That being said, TV viewing is a well established negative health behaviour, and these results have translational importance. With these limitations noted, the strengths of the present data mean that it has both scientific and practical implications.

\section{Relevance of findings to the field}

Diet: People with type 2 diabetes were more likely to report changing their diet in the past 5 years and less 
Table 2 Lifestyle characteristics of disease groups $(n=233110)$

\begin{tabular}{|c|c|c|c|c|}
\hline & \multicolumn{4}{|c|}{ Percentage within each disease group } \\
\hline & $\begin{array}{l}\text { No disease } \\
(n=103993)\end{array}$ & $\begin{array}{l}\text { CVD } \\
(n=113469)\end{array}$ & $\begin{array}{l}\text { Type } 2 \text { diabetes } \\
\text { without CVD }(n=4074)\end{array}$ & $\begin{array}{l}\text { Type } 2 \text { diabetes + } \\
\text { CVD }(n=11574)\end{array}$ \\
\hline \multicolumn{5}{|l|}{ Diet } \\
\hline Dietary change in past 5 years & 103902 & 113300 & 4070 & 11555 \\
\hline YES & 28.9 & 45.9 & 75.5 & 75.3 \\
\hline Meets fruit/veg guidelines & 102798 & 111554 & 3995 & 11347 \\
\hline YES & 29.8 & 32 & 35.7 & 36.6 \\
\hline 'Never eat' & 103848 & 113190 & 4039 & 11527 \\
\hline $\begin{array}{l}\text { Never eat sugar or foods/drinks containing } \\
\text { sugar }\end{array}$ & 14.8 & 21.0 & 49.8 & 51.2 \\
\hline \multicolumn{5}{|l|}{ Physical activity } \\
\hline Total physical activity* (MET.min/wk) & 103993 & 113469 & 4074 & 11574 \\
\hline$\leq 918$ (Low physical activity) & 25.0 & 30.5 & 35.4 & 40.1 \\
\hline$>918-1902$ & 25.0 & 24.2 & 22.5 & 22.2 \\
\hline$>1902-3706$ & 25.0 & 22.2 & 20.7 & 19.7 \\
\hline >3706-19 278 (high physical activity) & 25.0 & 23.2 & 21.3 & 18.0 \\
\hline Walking* (min/day) $^{*}$ & 103993 & 113469 & 4074 & 11574 \\
\hline 0-20 (low walking) & 31.5 & 33.9 & 36.6 & 40.4 \\
\hline $21-30$ & 20.8 & 20.4 & 21.0 & 19.6 \\
\hline $31-60$ & 26.7 & 25.8 & 23.0 & 23.6 \\
\hline $61-180$ & 21.1 & 19.9 & 19.4 & 16.3 \\
\hline Moderate activity* (min/day) & 103993 & 113469 & 4074 & 11574 \\
\hline 0-15 (low moderate) & 27.8 & 31.4 & 36.1 & 39.6 \\
\hline $16-30$ & 28.0 & 24.7 & 25.3 & 23.2 \\
\hline $31-60$ & 25.2 & 23.2 & 19.9 & 20.1 \\
\hline $61-180$ & 19.0 & 20.6 & 18.7 & 17.1 \\
\hline Vigorous activity* (min/day) & 103993 & 113469 & 4074 & 11574 \\
\hline 0 (low vigorous) & 34.2 & 46.1 & 49.7 & 56.5 \\
\hline $1-20$ & 20.3 & 19.6 & 19.7 & 18.0 \\
\hline $21-45$ & 21.7 & 16.7 & 14.5 & 13.1 \\
\hline $46-180$ & 23.7 & 17.6 & 16.1 & 12.3 \\
\hline $\begin{array}{l}\text { Meets UK government physical activity } \\
\text { guidelinest }\end{array}$ & 103993 & 113469 & 4074 & 11574 \\
\hline NO & 16.1 & 20.0 & 24.7 & 27.5 \\
\hline \multicolumn{5}{|l|}{ TV viewing } \\
\hline TV viewing* (h/day) & 103993 & 113469 & 4074 & 11574 \\
\hline$\leq 1$ (Low TV viewing) & 26.6 & 16.2 & 15.4 & 10.5 \\
\hline$>1-2$ & 30.5 & 24.5 & 22.3 & 19.3 \\
\hline$>2-3$ & 22.6 & 24.5 & 24.1 & 22.8 \\
\hline >3 (High TV viewing) & 20.3 & 34.8 & 38.1 & 47.3 \\
\hline \multicolumn{5}{|l|}{ Sleep } \\
\hline Sleep duration $\ddagger$ (h/night) & 103993 & 113469 & 4074 & 11574 \\
\hline$<7$ (Poor sleep duration) & 21.3 & 26.4 & 27.0 & 27.5 \\
\hline 7-8 (Good sleep duration) & 73.4 & 64.6 & 62.1 & 58.6 \\
\hline >8 (Poor sleep duration) & 5.3 & 9.1 & 10.8 & 13.9 \\
\hline \multicolumn{5}{|l|}{ Behavioural phenotype } \\
\hline $\begin{array}{l}\text { UNHEALTHY (low physical activity, high TV } \\
\text { viewing and poor sleep duration) }\end{array}$ & 1.8 & 5.2 & 5.8 & 10.0 \\
\hline $\begin{array}{l}\text { HEALTHY (high physical activity, low TV viewing } \\
\text { and good sleep duration) }\end{array}$ & 4.5 & 2.4 & 1.9 & 1.2 \\
\hline \multicolumn{5}{|c|}{$\begin{array}{l}\text { “For physical activity and TV viewing categories, quartiles were calculated from the 'No disease' group, so that their demarcators could be } \\
\text { applied to disease group. } \\
\text { tUK Government recommendations of } 150 \text { min of moderate or } 75 \text { min of vigorous activity per week. } \\
\text { †Physiological thresholds used rather than quartiles because the shape of the risk relationship is a U shape (not linear like physical activity } \\
\text { and TV viewing). } \\
\text { BMI, body mass index; CVD, cardiovascular disease. }\end{array}$} \\
\hline
\end{tabular}

likely to eat sugary foods compared with people with CVD or disease-free individuals. Dietary change is aligned to the current treatment advice for people with type 2 diabetes, ${ }^{24}$ and suggests that patients are acting on, or at least aware of, dietary advice. The food frequency questionnaire did not allow us to measure 


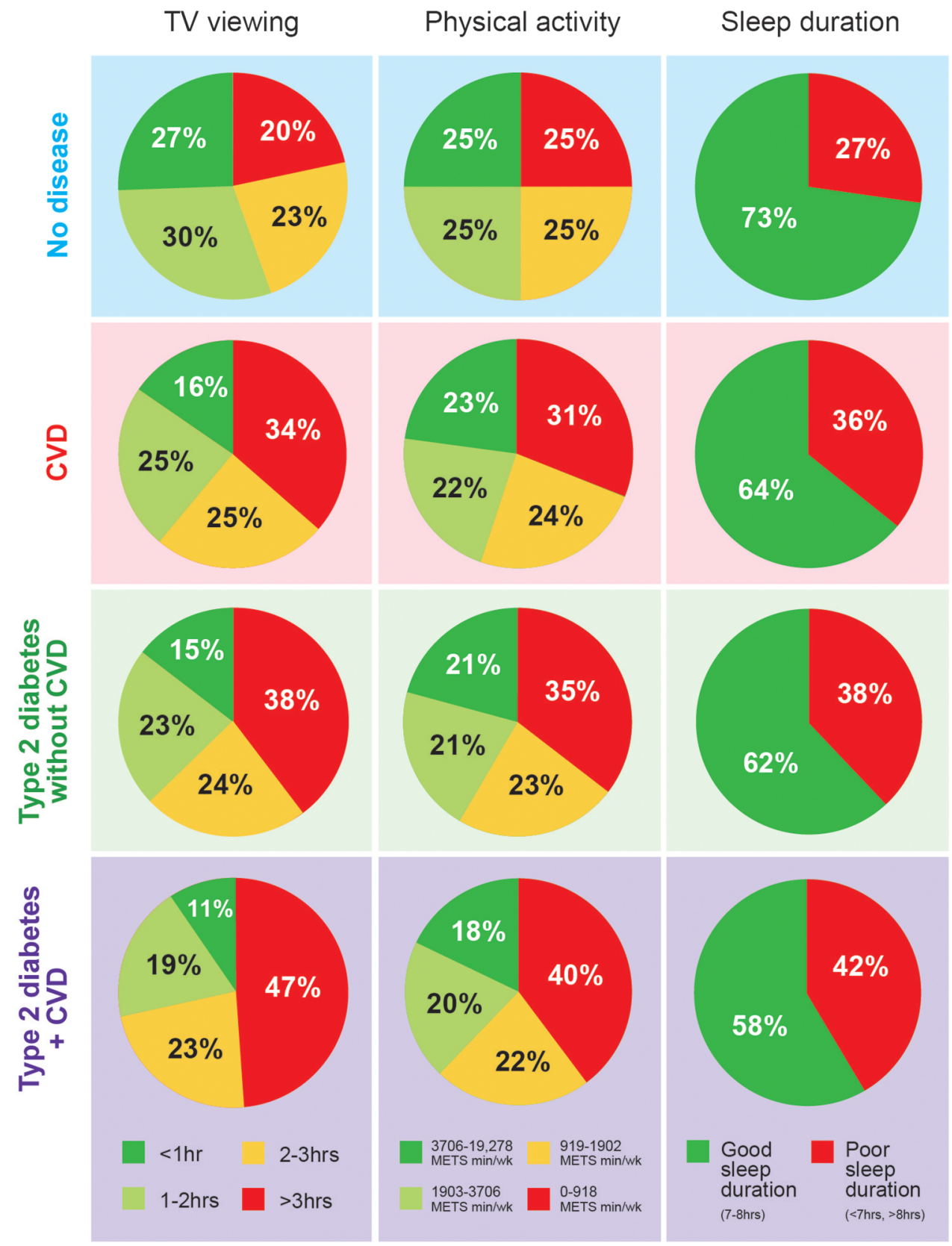

Figure 2 Distribution of physical activity, TV viewing and sleep duration in people with No disease, CVD, Type 2 diabetes without CVD, or Type 2 diabetes + CVD. Red indicates unhealthy and green indicates healthy lifestyle behaviours. CVD, cardiovascular disease.

energy intake; therefore, it is possible that, although participants had changed their diet, they ate more. To address excess calorie intake, we have controlled for BMI as a means to manage excess calorie intake. By contrast with those with type 2 diabetes, people with CVD consume more sugary foods than people with diabetes, and are less likely to have changed their diet, as advised by current advice. ${ }^{16}$ Cardioprotective diets are promoted to reduce cholesterol and blood pressure in CVD. ${ }^{25} 26$ Dietary advice remains the pillar of national guidelines for the management of type 2 diabetes, with evidence reporting that dietary changes can significantly improve glycemic control, ${ }^{27}$ and even reverse type 2 diabetes. ${ }^{28} 29$
Analysis of the self-report diet behaviour from the UK Biobank cohort suggests that national dietary messaging is reaching those with type 2 diabetes, but not those with CVD.

Physical activity: Those with cardiometabolic disease report less physical activity than healthy counterparts, with vigorous activity being the largest contributor to this difference. A recent prospective meta-analysis confirmed a dose-response relationship between physical activity and type 2 diabetes risk, with the strongest associations seen with vigorous activity, ${ }^{30}$ which along with our results suggests that a lack of vigorous activity plays a major role in cardiometabolic disease. Acute vigorous 
Table 3 OR $(95 \% \mathrm{Cl})$ of reporting low walking, low moderate and low vigorous activity across cardiometabolic disease

\begin{tabular}{llll}
\hline & Low walking (min/day) & $\begin{array}{l}\text { Low moderate } \\
\text { activity (min/day) }\end{array}$ & $\begin{array}{l}\text { Low vigorous } \\
\text { activity (min/day) }\end{array}$ \\
\hline No disease & 1.00 & 1.00 & 1.00 \\
CVD & $1.08(1.06$ to 1.10$)$ & $1.18(1.15$ to 1.20$)$ & $1.38(1.35$ to 1.40$)$ \\
Type 2 diabetes without CVD & $1.16(1.09$ to 1.24$)$ & $1.35(1.26$ to 1.45$)$ & $1.53(1.43$ to 1.63$)$ \\
Type 2 diabetes + CVD & $1.34(1.29$ to 1.40$)$ & $1.55(1.48$ to 1.61$)$ & $1.88(1.80$ to 1.96$)$ \\
\hline All models adjusted for age, gender, body mass index, sociodemographic (Townsend Deprivation Index and ethnicity), smoking, alcohol \\
and diet.
\end{tabular}

activity stimulates greater peripheral glucose uptake compared with non-vigorous activity, ${ }^{31}$ which may explain the stronger association with vigorous activity and cardiometabolic disease. National physical activity guidelines encourage individuals to perform at least 150 min of moderate activity or 75 min of vigorous activity weekly. ${ }^{13}$ Based on these recommendations, $16 \%$ of the 'No disease' group do not perform adequate physical activity levels, and the percentage rises with cardiometabolic disease.

$T V$ viewing: $\mathrm{TV}$ viewing was significantly higher in those with cardiometabolic disease compared with healthy adults. In a previous study, more than $3 \mathrm{~h}$ of TV viewing was strongly linked to all-cause mortality (RR 1.30; $95 \%$ CI 1.06 to 1.56$)^{6}$ suggesting that those in the highest quartile of TV viewing in the UK Biobank cohort are exposing themselves to detrimental health consequences. A strong evidence base is accumulating for the negative health consequences of TV viewing. It is likely that low muscle activity has a direct physiological health impact as skeletal muscle is the largest insulinsensitive organ in the body; however, TV viewing is also associated with other unhealthy behaviours such as snacking. ${ }^{23}$ As a result, it is consistently related to more adverse disease outcomes compared with daily sitting time and, therefore, should not be used as a surrogate marker for sedentary behaviour.

Sleep duration: These data reveal that across worsening cardiometabolic disease groups, the proportion of individuals reporting short or long sleep increases. We have defined optimal sleep duration as $7-8 \mathrm{~h}$ based on a recent review ${ }^{10}$ and our findings support previous observational studies which show a 'U-shaped' relationship between sleep duration and cardiometabolic disease. Sleep plays an integral role in metabolic regulation, ${ }^{32}$ with sleep restriction inducing insulin resistance and loss of circadian hormone changes. Indeed, the acute effects of sleep shortening are powerful. Sleep restricting healthy young men from $8 \mathrm{~h} /$ night to only $4 \mathrm{~h} /$ night for 1 week induces insulin resistance to a similar extent as people with type 2 diabetes. ${ }^{33}$ Sleep shortening also effects hormones that control appetite, ${ }^{34}$ elevating ghrelin and reducing leptin, which could explain the strong link between sleep deprivation, raised energy intake and weight gain. ${ }^{34}$ Persistent long sleep and increases in sleep duration over a 5 -year period have been linked to higher type 2 diabetes incidence. ${ }^{35}$ However, the physiological impact of long sleep is yet to be fully understood. Although there will be a clear impact of long sleep on the opportunity to be physically active during wakefulness, more work is needed to explore the impact of normalising sleep in people who have long sleep. The benefits of improving sleep in people with existing cardiometabolic disease remains poorly described. However, given the potent effects of sleep on physiological function, these data highlight poor sleep as a potential therapeutic target in people with CVD and/or type 2 diabetes.

Clustered lifestyle behaviours: The results from this large population-based study also indicate an increased likelihood of reporting an 'unhealthy phenotype' encompassing low physical activity, high TV viewing and poor sleep duration, across worsening cardiometabolic disease groups. The 'Type 2 diabetes + CVD' group, who have a

Table 4 OR $(95 \% \mathrm{Cl})$ of reporting low physical activity, high sitting time, poor sleep and all behaviours combined across cardiometabolic disease

\begin{tabular}{lllll}
\hline & $\begin{array}{l}\text { Low physical } \\
\text { activity }\end{array}$ & High TV viewing & Poor sleep & $\begin{array}{l}\text { Low physical activity + } \\
\text { high sitting+poor sleep }\end{array}$ \\
\hline No disease & 1.00 & 1.00 & 1.00 & 1.00 \\
CVD & $1.23(1.20$ to 1.25$)$ & $1.42(1.39$ to1.45) & $1.37(1.34$ to1.39) & $2.15(2.03$ to 2.28$)$ \\
Type 2 diabetes without CVD & $1.43(1.34$ to 1.53$)$ & $1.59(1.49$ to1.69) & $1.38(1.30$ to1.47) & $2.14(1.85$ to 2.48$)$ \\
Type 2 diabetes + CVD & $1.71(1.64$ to 1.78) & $1.92(1.85$ to 1.99) & $1.52(1.46$ to1.58) & $3.29(3.02$ to 3.58$)$ \\
\hline All models adjusted for age, gender, body mass index, sociodemographic (Townsend Deprivation Index and ethnicity), smoking, alcohol \\
$\begin{array}{llll}\text { and diet. } \\
\text { CVD, cardiovascular disease. }\end{array}$
\end{tabular}


Figure 3 Radar chart showing the proportion of adults in each group who were categorised as physical activity or TV viewing, or 'good' or 'poor' for sleep duration. Green side indicates healthy non-diet lifestyle behaviours, whereas red side indicates unhealthy non-diet behaviours. There is a shift leftwards towards unhealthy behaviours with cardiometabolic disease. either 'high' or 'low' for total

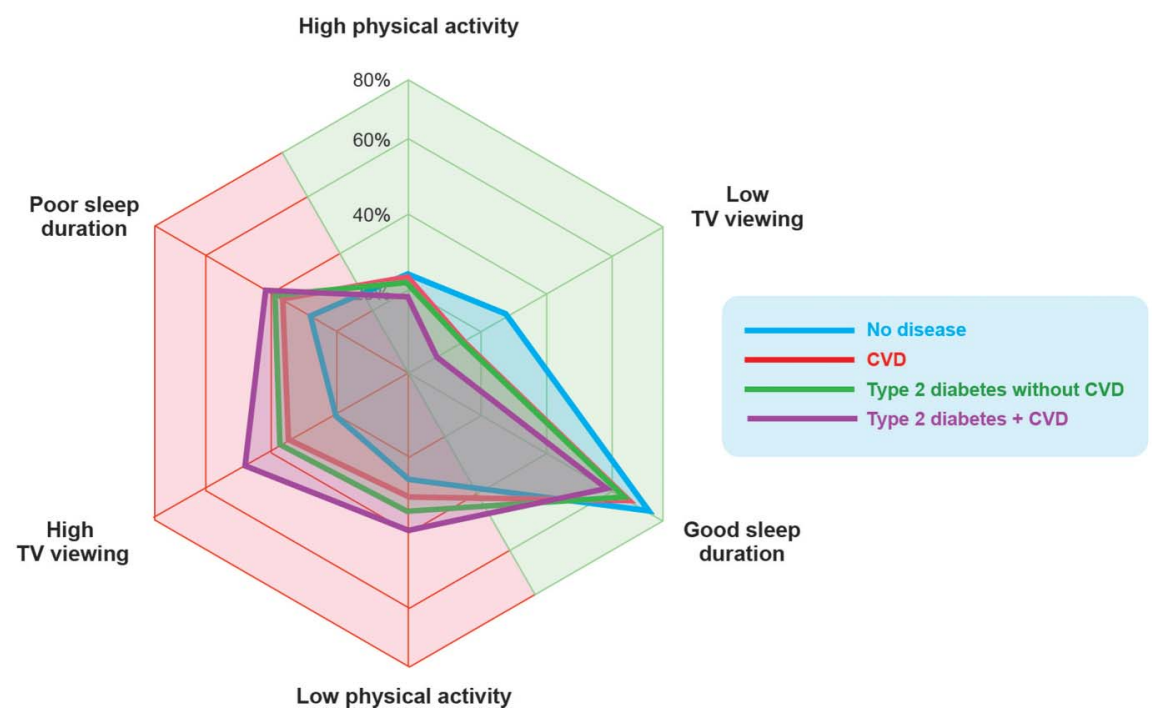

particularly poor prognosis, were more likely to report low physical activity, high TV viewing and poor sleep duration compared with all other groups, suggesting that these non-diet lifestyle behaviours may be exposing individuals to greater cardiometabolic risk beyond their disease. In the context of cardiometabolic disease and obesity, it is becoming increasingly common to combine physical activity and sitting as a joint association. ${ }^{36}{ }^{37} \mathrm{We}$ have added sleep into our analysis, as we propose that all three behaviours are interdependent in their influence on metabolic control. Indeed, the clustering of these behaviours produces higher odds with cardiometabolic disease compared with individual behaviours. During sleep, there is a reduction in glucose utilisation, with an overall rise in plasma glucose. ${ }^{38}$ By contrast, throughout waking hours, physical activity stimulates peripheral glucose uptake, ${ }^{39}$ and is important for maintenance of euglycemia. Physical activity may be viewed as an activator of metabolism, whereas sleep is vital for restoring and resetting homeostasis, largely through energy regulation and repair. A lack of activation or restoration results in insulin resistance, a prominent feature of cardiometabolic disease. ${ }^{40}$ Individually, low physical activity, high TV viewing and poor sleep duration have negative metabolic consequences. The present data suggest that people with CVD and/or type 2 diabetes are more likely to be exposed to a potent negative 'behavioural phenotype' consisting of low physical activity, high TV viewing and poor sleep duration simultaneously.

\section{Implications for care teams, policymakers and people with cardiometabolic disease}

Data from the UK Biobank suggest that poor non-diet lifestyle behaviours are prominent and unaddressed behaviours in the prevention and management of cardiometabolic disease. Our findings should not be taken to understate the importance of diet in cardiometabolic health. A balanced diet and weight management are critical, and efforts should continue to support people accordingly. However, the government recently described physical activity as a 'key health priority in its own right' highlighting the importance of strategic planning with various sectors spanning transport, infrastructure and training of healthcare professionals. In 2014, Public Health England produced a framework to embed physical activity into the fabric of daily life, ${ }^{42}$ and the first national NHS prevention programme designed to prevent type 2 diabetes through diet and physical activity interventions. ${ }^{43}$ The present data reinforces the pressing need for evidence-based and effective programmes for physical activity for people with CVD and type 2 diabetes.

Awareness of the importance of sedentary behaviours in chronic disease lags physical activity, but is growing rapidly. In 2010, the department of health and the sedentary behaviour and expert working group recommended that more emphasis needed to be placed on minimising time spent sedentary. ${ }^{44}$ Indeed, NICE guidelines for type 2 diabetes prevention note the importance of reducing sitting time. ${ }^{15}$ Cross-sectional and longitudinal data provide evidence for the importance of TV viewing in cardiometabolic health, ${ }^{6}{ }^{45} 46$ yet the importance of daily sitting time, which is a better marker of sedentary behaviour, is in question. ${ }^{47}$ Before guidelines and policies can be targeted towards sedentary behaviour, well-controlled intervention studies are urgently required to define the role of daily sitting in cardiometabolic disease. By contrast with physical activity and sitting, NICE guidelines for CVD and type 2 diabetes do not comment on sleep, despite the present data revealing that one in three of people with CVD, and nearly half the people with type 2 diabetes, sleep either too much or too little. Despite strong cross-sectional data, evidence is needed to define the impact of helping people with cardiometabolic disease achieve good sleep, to help inform guidelines and policy in this area.

A major finding from the present data was the clustering of physical activity, TV viewing and sleep behaviour. This is important as, to date, intervention studies have focused on changing a single lifestyle behaviour, with very few targeting multiple lifestyle behaviours. ${ }^{48}$ 
Given the clustering of these non-diet lifestyle behaviours, exploration of interventions incorporating physical activity, TV viewing and sleep, together may add value and should be the focus of future policies and programmes.

In summary, the preset data demonstrates that those with more advanced cardiometabolic disease undertake too little physical activity, have high TV viewing times and poor sleep duration, yet report important positive dietary changes within the past 5 years. These non-diet lifestyle behaviours are clustered, and indeed those with the worst cardiometabolic disease are three times more likely to display an 'unhealthy behavioural phenotype' compared with disease-free individuals, independent of age, gender, BMI and socioeconomic status. These novel data highlight that there remains a significant unaddressed behavioural phenotype of CVD and type 2 diabetes that places these people at excess risk of worsening cardiometabolic health. Strategies are urgently required to address physical activity, TV viewing and sleep to assist patients, care teams and policymakers in making effective decisions for the management and prevention of CVD and type 2 diabetes.

\author{
Author affiliations \\ ${ }^{1}$ Faculty of Medical Sciences, Institute of Cellular Medicine, Newcastle \\ University, Newcastle upon Tyne, UK \\ ${ }^{2}$ Prevention Research Collaboration, Sydney School of Public Health, Charles \\ Perkins Centre D17, University of Sydney, New South Wales, Australia \\ ${ }^{3}$ Faculty of Medical Sciences, Institute of Neuroscience, Newcastle University, \\ Newcastle upon Tyne, UK
}

Acknowledgements This research was conducted using the UK Biobank resource. The authors would like to thank the UK Biobank participants and investigators for making this study possible. We would also like to acknowledge the Newcastle University Centre for Ageing and Vitality sponsored by the BBSRC, EPSRC, ESRC and MRC.

Contributors $S C, J Y C$ and $A B$ performed data analysis and epidemiology expertise was provided by JYC and $A B$. Data interpretation and statistical input was provided by JYC, MC, AB and MIT. SC and MIT wrote the first draft of the paper and all other authors contributed to successive drafts. All authors read and approved the final manuscript. All authors have approved the final report. All authors had full access to the data in the study and take responsibility for its integrity and the accuracy of the data analysis. MIT is the guarantor of this work.

Funding The UK Biobank was established by the Wellcome Trust medical charity, Medical Research Council, Department of Health, Scottish Government and the Northwest Regional Development Agency. It has also received funding from the Welsh Assembly Government and the British Heart Foundation. The analyses were supported by the Newcastle University Centre for Ageing and Vitality sponsored by the BBSRC, EPSRC, ESRC and MRC; JYC was supported by a Postdoctoral Fellowship (number 100567) from the National Heart Foundation of Australia; MIT was supported by a Senior Research Fellowship from the National Institute for Health Research (NIHR-SRF-04-017).

Competing interests None declared.

Ethics approval The UK Biobank study was approved by the North West Multi-centre Research Ethics Committee, the England and Wales Patient Information Advisory Group, and the Scottish Community Health Index Advisory Group.

Provenance and peer review Not commissioned; externally peer reviewed.
Data sharing statement No additional data are available.

Open Access This is an Open Access article distributed in accordance with the terms of the Creative Commons Attribution (CC BY 4.0) license, which permits others to distribute, remix, adapt and build upon this work, for commercial use, provided the original work is properly cited. See: http:// creativecommons.org/licenses/by/4.0/

\section{REFERENCES}

1. BHF Cardiovascular Disease Statistics UK Factsheet [internet]. British Heart Foundation. 2015. https://www.bhf.org.uk/research/ heart-statistics

2. Sarwar N, Gao P, Seshasai SRK, et al. Diabetes mellitus, fasting blood glucose concentration, and risk of vascular disease: a collaborative meta-analysis of 102 prospective studies. Lancet 2010;375:2215-22.

3. State of the nation (England): challenges for 2015 and beyond [internet]. Diabetes UK. 2015. https://www.diabetes.org.uk/About_us/ What-we-say/Statistics/State-of-the-nation-challenges-for-2015-andbeyond/

4. Hex N, Bartlett C, Wright $D$, et al. Estimating the current and future costs of type 1 and type 2 diabetes in the UK, including direct health costs and indirect societal and productivity costs. Diabet Med 2012;29:855-62.

5. [No authors listed]. Diabetes mellitus: a major risk factor for cardiovascular disease. A joint editorial statement by the American Diabetes Association; The National Heart, Lung, and Blood Institute; the Juvenile Diabetes Foundation International; The National Institute of Diabetes and Digestive and Kidney Diseases; and The American Heart Association. Circulation 1999;100:1132-3.

6. Grøntved A, Hu FB. Television viewing and risk of type 2 diabetes, cardiovascular disease, and all-cause mortality: a meta-analysis. JAMA 2011;305:2448-55.

7. Bell JA, Hamer M, Batty GD, et al. Combined effect of physical activity and leisure time sitting on long-term risk of incident obesity and metabolic risk factor clustering. Diabetologia 2014;57:2048-56.

8. Murray CJ, Richards MA, Newton JN, et al. UK health performance: findings of the Global Burden of Disease Study 2010. Lancet 2013;381:997-1020.

9. Liu Y, Wheaton AG, Chapman DP, et al. Sleep duration and chronic diseases among US adults age 45 years and older : evidence from the 2010 Behavioral Risk Factor Surveillance System. Sleep 2013;36:1421-7.

10. Shan Z, Ma H, Xie M, et al. Sleep duration and risk of type 2 diabetes: a meta-analysis of prospective studies. Diabetes Care 2015;38:529-37.

11. WHO. Global strategy on diet, physical activity and health. Geneva: World Health Organisation, 2004.

12. Bull $\mathrm{F}$, Milton $\mathrm{K}$, Kahlmeier $\mathrm{S}$, et al. Turning the tide: national policy approaches to increasing physical activity in seven European countries. Br J Sports Med 2015;49:749-56.

13. Physical activity guidelines in the UK: review and recommendations [internet]. Department of Health. 2011. https://www.gov.uk/ government/publications/uk-physical-activity-guidelines

14. Your guide to the eatwell plate booklet [internet]. Public Health England. 2014. https://www.gov.uk/government/publications/theeatwell-plate-how-to-use-it-in-promotional-material

15. Type 2 diabetes: prevention in people at high risk [internet]. National Institute for Health and Care Excellence. 2012. https://www.nice.org. uk/guidance/ph38

16. Cardiovascular disease: risk assessment and reduction, including lipid modification [internet]. National Institute for Health and Care Excellence. 2014. https://www.nice.org.uk/guidance/cg181

17. Rationale, design and development of a large-scale prospective resource [internet]. UK Biobank. 2007. http://www.ukbiobank.ac.uk/ resources/

18. International statistical classification of diseases and related health problems 10th revision [internet]. World Health Organisation. http:// www.who.int/classifications/icd/en/

19. Craig $C L$, Marshall AL, Sjöström M, et al. International physical activity questionnaire: 12 -country reliability and validity. Med Sci Sports Exerc 2003;35:1381-95.

20. IPAQ. Guidelines for Data Processing and Analysis of the International Physical Activity Questionnaire (IPAQ) - Short and Long Forms. 2005:1-15.

21. Hu FB, Li TY, Colditz GA, et al. Television watching and other sedentary behaviors in relation to risk of obesity and type 2 diabetes mellitus in women. JAMA 2003;289:1785-91. 
22. Willett WC, Sampson L, Stampfer MJ,, et al. Reproducibility and validity of a semiquantitative food frequency questionnaire. Am J Epidemiol 1985;122:51-65.

23. Cleland VJ, Schmidt MD, Dwyer T, et al. Television viewing and abdominal obesity in young adults: is the association mediated by food and beverage consumption during viewing time or reduced leisure-time physical activity? Am J Clin Nutr 2008;87:1148-55.

24. NICE. Type 2 diabetes in adults: management of type 2 diabetes in adults. Draft for consultation, 2015.

25. Kris-Etherton PM. Fish consumption, fish oil, omega-3 fatty acids, and cardiovascular disease. Circulation 2002;106:2747-57.

26. de Lorgeril M, Salen P, Martin JL, et al. Mediterranean diet, traditional risk factors, and the rate of cardiovascular complications after myocardial infarction: final report of the Lyon Diet Heart Study. Circulation 1999;99:779-85.

27. Brand-Miller J, Hayne S, Petocz P, et al. Low-glycemic index diets in the a meta-analysis of randomized controlled trials. Diabetes Care 2003;26:2261-7.

28. Lim EL, Hollingsworth KG, Aribisala BS, et al. Reversal of type 2 diabetes: normalisation of beta cell function in association with decreased pancreas and liver triacylglycerol. Diabetologia 2011;54:2506-14.

29. Andrews RC, Cooper AR, Montgomery AA, et al. Diet or diet plus physical activity versus usual care in patients with newly diagnosed type 2 diabetes: the Early ACTID randomised controlled trial. Lancet 2011;378:129-39.

30. Aune D, Norat T, Leitzmann M, et al. Physical activity and the risk of type 2 diabetes: a systematic review and dose-response meta-analysis. Eur J Epidemiol 2015;30:529-42.

31. Mayer-Davis EJ, D'Agostino R Jr, Karter AJ, et al. Intensity and amount of physical activity in relation to insulin sensitivity: the Insulin Resistance Atherosclerosis Study. JAMA 1998;279:669-74.

32. Trenell MI, Marshall NS, Rogers NL. Sleep and metabolic control: waking to a problem? Clin Exp Pharmacol Physiol 2007;34:1-9.

33. Spiegel K, Leproult R, Van Cauter E. Impact of sleep debt on metabolic and endocrine function. Lancet 1999;354:1435-9.

34. Taheri S, Lin L, Austin D, et al. Short sleep duration is associated with reduced leptin, elevated ghrelin, and increased body mass index. PLoS Med 2004;1:e62.

35. Ferrie JE, Kivimäki M, Akbaraly TN, et al. Change in sleep duration and type 2 diabetes: the Whitehall II Study. Diabetes Care 2015;38:1467-72.

36. Chu AH, Moy FM. Joint association of sitting time and physical activity with metabolic risk factors among middle-Aged Malays in a developing country: a cross-sectional study. PLOS ONE 2013;8: e61723.

37. Petersen CB, Nielsen AJ, Bauman A, et al. Joint association of physical activity in leisure and total sitting time with metabolic syndrome amongst 15,235 Danish adults: a cross-sectional study. Prev Med 2014;69:5-7.

38. Van Cauter E, Polonsky KS, Scheen AJ. Roles of circadian rhythmicity and sleep in human glucose regulation. Endocr Rev 1997;18:716-38.

39. Hayashi T, Wojtaszewski J, Goodyear LJ. Exercise regulation of glucose transport in skeletal muscle. Am J Physiol 1997;273(6 Pt 1): E1039-51.

40. Ferrannini E, Haffner SM, Mitchell BD, et al. Hyperinsulinaemia: the key feature of a cardiovascular and metabolic syndrome. Diabetologia 1991;34:416-22.

41. Government response to the HSC report on the Impact of physical activity and diet on health (web version) [internet]. Department of Health. 2015. https://www.gov.uk/government/publications/impact-ofphysical-activity-and-diet-on-health

42. Everybody active, every day: an evidence-based approach to physical activity [internet]. Public Health England. 2014. https://www. gov.uk/government/publications/everybody-active-every-day-aframework-to-embed-physical-activity-into-daily-life

43. NHS diabetes prevention programme (NHS DPP) non-diabetic hyperglycemia [internet]. Public Health England. 2015. https://www. gov.uk/government/publications/nhs-diabetes-preventionprogramme-non-diabetic-hyperglycaemia

44. The Sedentary Behaviour and Obesity Expert Working Group. Sedentary behaviour and obesity: review of the current scientific evidence [internet]. Public Health England. 2010. https://www.noo. org.uk/Resources/DH_publications

45. Wijndaele K, Healy GN, Dunstan DW, et al. Increased cardiometabolic risk is associated with increased TV viewing time. Med Sci Sports Exerc 2010;42:1511-18.

46. Smith L, Hamer M. Television viewing time and risk of incident diabetes mellitus: the English Longitudinal Study of Ageing. Diabet Med 2014;31:1572-6.

47. Pulsford RM, Stamatakis E, Britton AR, et al. Associations of sitting behaviours with all-cause mortality over a 16-year follow-up: the Whitehall II study. Int J Epidemiol 2015;44: 1909-16.

48. King K, Meader N, Wright $\mathrm{K}$, et al. Characteristics of interventions targeting multiple lifestyle risk behaviours in adult populations: a systematic scoping review. PLOS ONE 2015;10:e0117015. 